\title{
Crude Oil Fouling in Heat Exchangers: A Study on Effects of Influencing Forces
}

\author{
Sampath Emani ${ }^{1}$, M. Ramasamy ${ }^{1 *}$, Ku Zilati Ku Shaari ${ }^{1}$ \\ ${ }^{1}$ Chemical Engineering Department, Universiti Teknologi PETRONAS, 32610 Bandar Seri Iskandar, Perak D.R., Malaysia
}

\begin{abstract}
One of the major concerns in petroleum refinery preheat trains is identified as fouling. Fouling impacts the refinery economics and environment heavily. Various approaches to mitigate fouling have not yielded the desired results. This is due to lack of understanding on the effect of influencing forces on crude oil fouling in heat exchangers. Therefore, this study attempts to investigate the effects of various forces such as gravity, Saffman Lift, drag and thermophoretic on crude oil fouling in heat exchangers through Computational Fluid Dynamics (CFD) simulations. From the simulations, it is observed that the higher particle size and particle concentration resulted in higher deposition of particles. Deposition velocities increase for larger sized particles and decrease for small and medium sized particles. The Increased flow velocities and surface roughness increases wall shear and mitigate fouling. Lower temperature gradients at the heat exchanger surface decreases deposition rates due to high thermophoretic forces. The mass deposition rate is reduced by 10.3 and $16.9 \%$ with 0.03 and $0.05 \mathrm{~Pa}$, respectively, for $0.14 \mathrm{~m} / \mathrm{s}$ flow velocity. Also, the mass deposition rate is reduced by 15.6 and $25.1 \%$ with 0.03 and $0.05 \mathrm{~Pa}$, respectively, for 0.47 $\mathrm{m} / \mathrm{s}$ flow velocity. With increased surface roughness from 0.03 to $0.05 \mathrm{~mm}$, the mass deposition rate is reduced by 11.48 and $19.18 \%$, respectively, for $0.14 \mathrm{~m} / \mathrm{s}$ flow velocity. Also, for $0.47 \mathrm{~m} / \mathrm{s}$ flow velocity, the mass deposition rate is reduced by 18.84 and $32.92 \%$ for 0.03 - and $0.05-\mathrm{mm}$ surface roughness, respectively.
\end{abstract}

\section{Introduction}

The impacts of fouling, its mechanisms and the phenomena has been well known globally. However, the physical and chemical aspects involved in the predominated fouling mechanisms (particulate and chemical reaction fouling) have not been well understood. This is due to the fact that fouling experiments are generally tedious to perform, timeconsuming and costly. Fouling in crude preheat train heat exchangers in refineries is a complex phenomenon [1-3].

The particles distribution and pressure drop in a liquidsolid fluidized bed heat exchanger was studied through CFD simulations [4]. Volume-of-Fluid based Eulerian multiphase fluid model was used to analyse the flow of liquid-solid in the heat exchanger. From the simulations, it is noticed that due to incomplete fluidization, more particles are deposited in the lower tubes of the horizontal tube bundle. Also, uniformity distribution of particles and high dynamic pressure drops were obtained in high velocity regions. Most of the particles that are deposited are observed to be of larger diameter. Therefore, large diameter particles would not be recommended in the operation of horizontal tube bundle heat exchanger.

The Eulerian-Lagrangian approach for multi-phase flow modelling has been widely used to ease the understanding of the particles transport in a flow domain [5-11]. The computational time for the simulations with Eulerian-Lagrangian approach is high for fluids composed with a high volume of particles. Despite the difficulties with the computational time and power, the Eulerian-Lagrangian approach remains the most considered model to solve the fluid flow modelling involving multi-phases [12].

However, the CFD studies performed in shell and tube heat exchangers, as reported in literature, did not consider the existence of asphaltenes particles in the bulk fluid as a discrete phase. The discrete-phase models have the capability to analyze the particulate and chemical reaction fouling routes on the heat transfer surfaces, but these simulation models have not been well utilized to understand the fouling phenomena of crude oils. The limited fundamental understanding of its causes and mechanisms led the fouling phenomena as a long-standing problem. Better understanding of its phenomena can help to assist development of appropriate mitigation strategies $[13,14]$.

\section{Fouling Phenomena Methodology using CFD}

In the present study, a multi-pass shell and tube heat exchanger with triangular tube pitch arrangement is considered. The details of the heat exchanger geometry, validation and material properties are shown in our previously published manuscript [15]. The fouling phenomena investigated in the heat exchanger geometries are based on asphaltenes deposition (particulate) fouling. The fouling phenomena is studied only through CFD simulations. The fouling fluid 
medium is crude oil and temperature-dependent correlations are invoked as user defined functions in the CFD software for material properties of crude oil. The fouling fluid is assumed to contain the solid particles called as asphaltenes. The non-fouling fluid medium is assumed to have similar physical and thermal properties as crude oil but does not comprise of any solid particles. As fouling is a dynamic process, the CFD simulations with fouling precursors are performed in a timedependent iterative process. The fouling phenomena in CFD is studied through Eulerian-Lagrangian approach. Asphaltenes particles are already present in the bulk fluid and precipitation from bulk fluid is neglected. The particle maintains a uniform size and does not grow once entered the fluid domain. The stability and consistency of the simulation results are considered as the convergence criteria for the CFD simulations. The validation of the CFD model is performed for steadystate fluid flow analysis without fouling precursors and un-steady fluid flow analysis with fouling precursors. The tube-side heat transfer coefficients are validated with Colburn correlation and the shell-side heat transfer coefficients are validated with Bell-Delaware correlation for steady-state fluid flow analysis without fouling precursors. The fouling data obtained from the time dependent CFD simulations are validated with existing literature fouling data. The fluid-flow operating conditions such as bulk velocity, temperature gradients, wall shear and surface roughness are varied to study the fouling mitigation techniques in the heat exchangers.

The initial step of CFD analysis is to formulate the flow problem. With the available computational power and time, precise CFD simulations are possible to perform to improve the understanding of the fluid-flow in processing equipment. The geometry about which fluidflow is to be analysed requires modelling. The geometry modelling is performed using a CAD software package, Ansys ICEM-CFD. The geometry is modelled in such a manner as to provide input for the mesh generation. The fluid flow domain is discretized into small numerical grids on which governing equations will be applied. However, the grid blocks may be overlapping, contiguous and abutting. Therefore, the grid is maintained above the minimal grid quality by measures of relative grid spacing and grid skewness to ensure the quality of the grid. Once the mesh is generated and mesh independence test is performed, the boundary conditions on the surfaces of the geometry are specified. The boundary conditions include the inlets, wall and outlet conditions. The basic governing equations on the cartesian coordinates related to fluid dynamics are provided in this section. However, based on the requirements, cylindrical coordinates governing equations are also used. The operating conditions are then defined on boundaries of the fluid flow domain. The choice of turbulence, non-relaxation factors and spatial discretization schemes were reviewed thoroughly, and appropriate models were considered for performing the simulations. The convergence residuals were maintained at minimum 1e-04 with second order discretization. With the iterative process, the solution was continuously monitored till the converged solution has been achieved. The fluid-flow and heat transfer processes are generally governed by various differential equations as stated below:

Continuity equation:

$\frac{\partial \rho}{\partial t}+\nabla \cdot(\overline{\rho v})=0$

Momentum equation:

$\frac{\partial(\overline{\rho v)}}{\partial t}+\nabla \cdot(\overline{\rho v v)}=-\nabla p+\nabla \cdot(\bar{\tau})+\rho \bar{g}$

Energy equation:

$\frac{\partial\left(\rho C_{P} T\right)}{\partial t}+\nabla \cdot\left(\rho C_{P} \vec{v} T\right)=\nabla \cdot(k \Delta T)+H$

The dimensions of the heat exchanger are given in Table 1. The shell and tube exchanger consist of four major parts, the geometry of which is shown in Figure 1. The mesh of the shell and tube heat exchanger consists of 2.8 million tetrahedral cells as shown in Figure 2.

Table 1. Heat exchanger dimensions

\begin{tabular}{|c|c|}
\hline Description & Value \\
\hline Shell diameter $(\mathrm{mm})$ & 154.05 \\
\hline Tube wall thickness $(\mathrm{mm})$ & 1.65 \\
\hline No. of tubes & 10 \\
\hline Tube outside diameter $(\mathrm{mm})$ & 21.34 \\
\hline $\begin{array}{c}\text { Inlet, outlet nozzles diameter } \\
(\mathrm{mm})\end{array}$ & 26.645 \\
\hline Tube length $(\mathrm{mm})$ & 1000 \\
\hline Baffle spacing $(\mathrm{mm})$ & 50.8 \\
\hline No. of baffles & 16 \\
\hline Baffle cut $(\%)$ & 29 \\
\hline
\end{tabular}

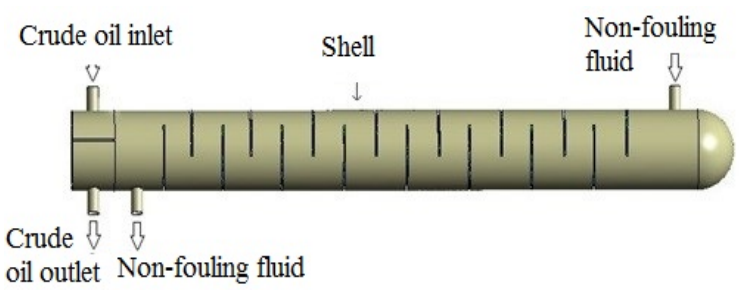

Fig. 1. Shell and tube heat exchanger CAD model

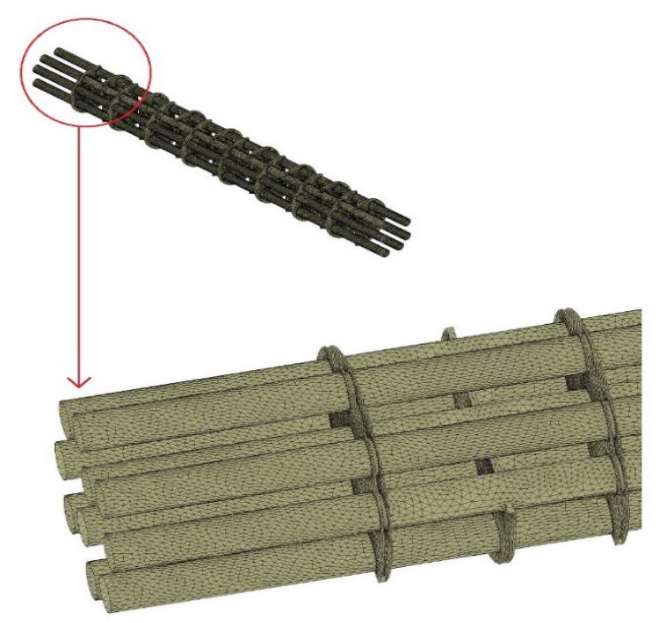

Fig. 2. Mesh of shell and tube heat exchanger 
Table 2. Simulation runs for multi-pass shell and tube heat exchanger with shell side bulk temperature as $441 \mathrm{~K}$

\begin{tabular}{|c|c|c|}
\hline Simulation run & $\begin{array}{c}\text { Flow- } \\
\text { velocity (m/s) }\end{array}$ & $\begin{array}{c}\text { Tube-side bulk } \\
\text { temperature (K) }\end{array}$ \\
\hline 1 & 0.5 & 335 \\
\hline 2 & 1.0 & 335 \\
\hline 3 & 1.5 & 335 \\
\hline 4 & 2.0 & 335 \\
\hline 5 & 0.5 & 355 \\
\hline 6 & 1.0 & 355 \\
\hline 7 & 1.5 & 355 \\
\hline 8 & 2.0 & 355 \\
\hline 9 & 0.5 & 375 \\
\hline 10 & 1.0 & 375 \\
\hline 11 & 1.5 & 375 \\
\hline 12 & 2.0 & 375 \\
\hline
\end{tabular}

\section{CFD Simulations Results and Discussions}

The asphaltenes deposition along the tube for different particle sizes and fluid velocities are shown in Figures 3 and 4, respectively. The gravitational, lift, thermophoretic and drag forces acing on the particles along the tube are shown in Figures 5-8, respectively and In tube pass 1, the gravitational and drag forces increased along the tube, the lift and thermophoretic forces decreased along the tube. Among all the forces, the gravitational force acting on the asphaltenes particles is higher, the drag and thermophoretic forces are observed to have less impact on the asphaltenes particles. As the gravitational force is observed to be the dominating force, the asphaltenes deposition along the tube followed the same trend as the gravitational force. In tube pass 2, the gravitational, thermophoretic and drag forces decreased along the tube and the lift force increased along the tube. Among all the forces, the gravitational force acting on the asphaltenes particles is observed to be high, the drag and thermophoretic forces are observed to have less impact on the asphaltenes particles. As the gravitational force is observed to be the dominating force, the asphaltenes deposition along the tube followed the same trend as the gravitational force.

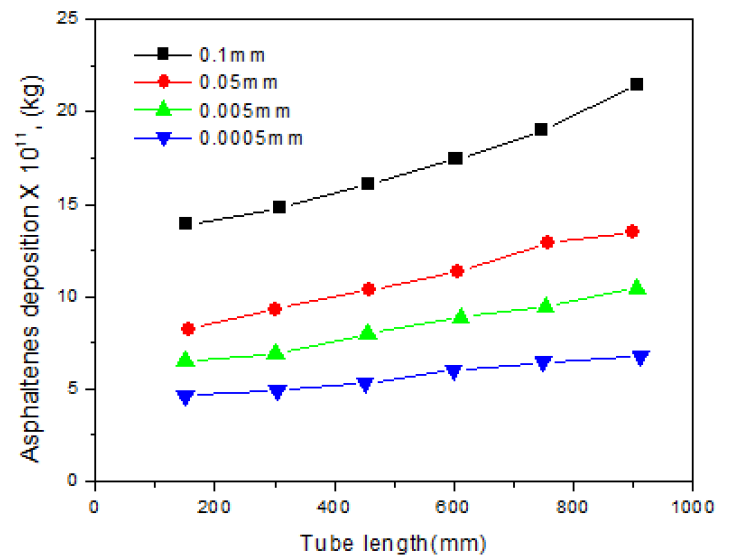

(a)

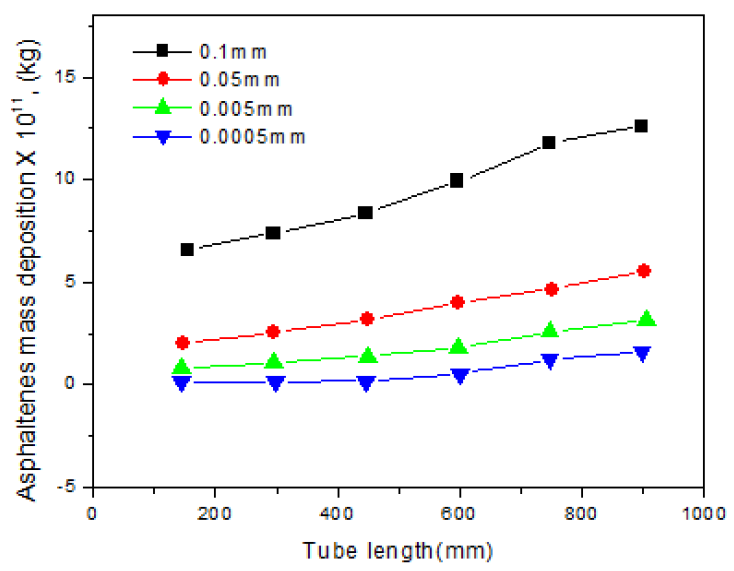

(b)

Fig. 3. Asphaltenes deposition across the tube passes (a) 1 and (b) 2

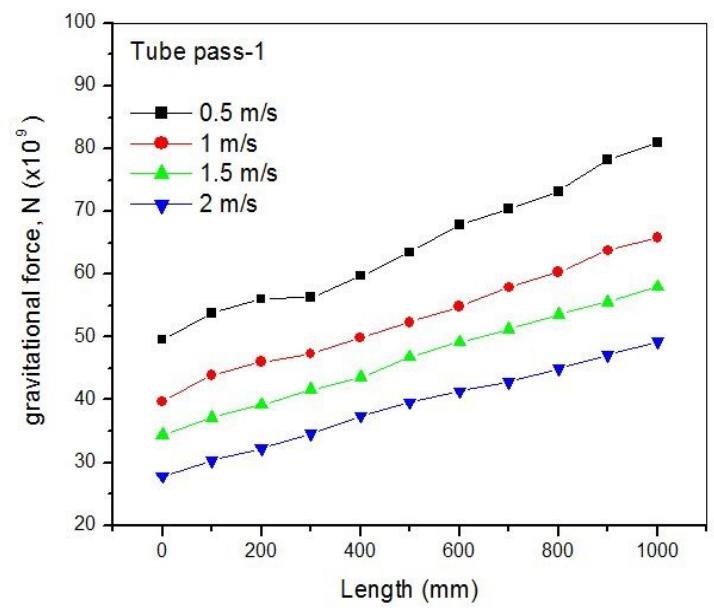

(a) 


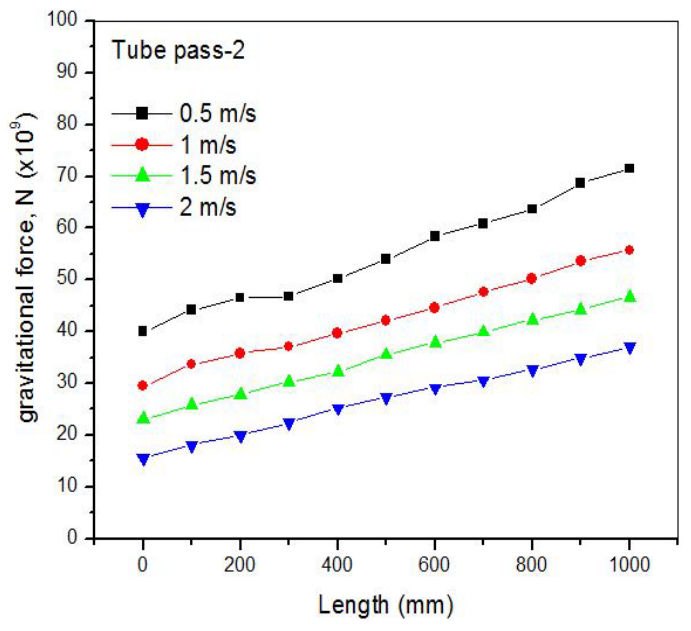

(b)

Fig. 4. Gravitational force vs tube length across the tube passes (a) 1 and (b) 2

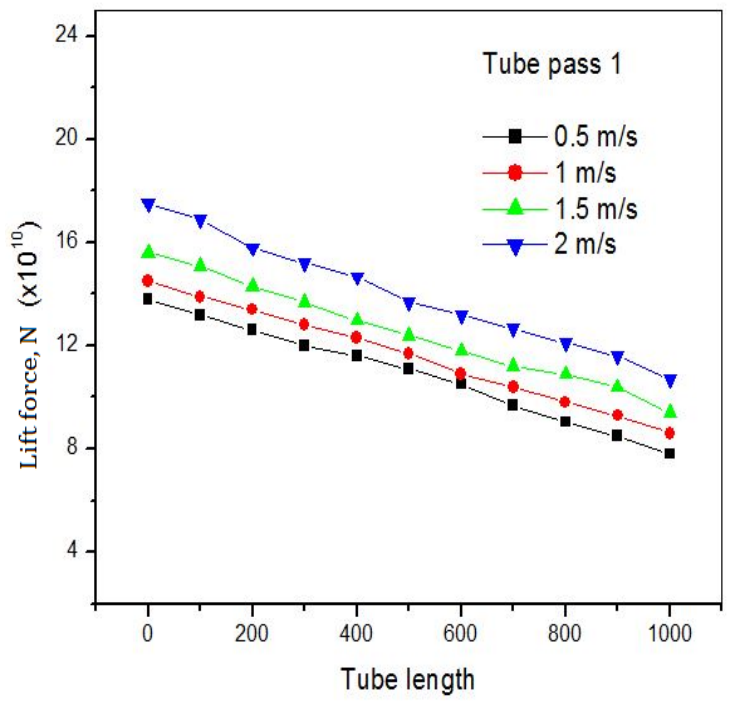

(a)

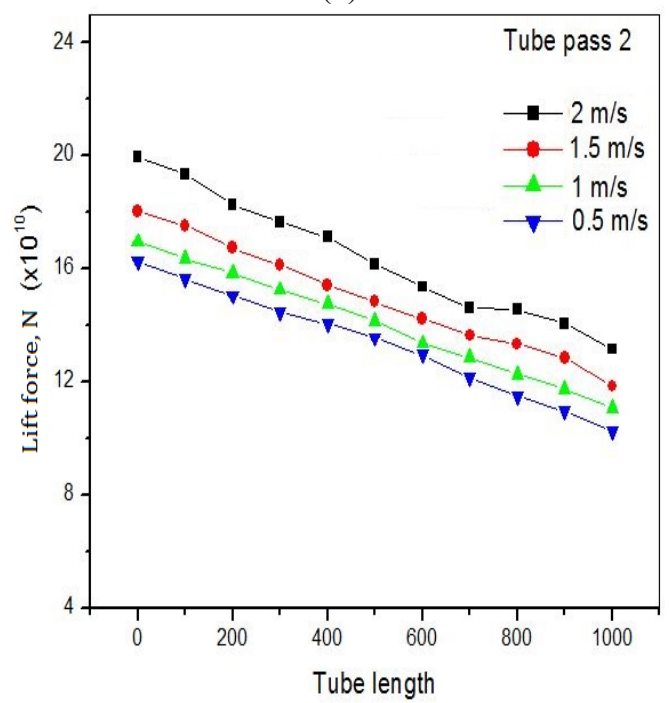

(b)
Fig. 5. Lift force vs tube length across the tube passes (a) 1 and (b) 2

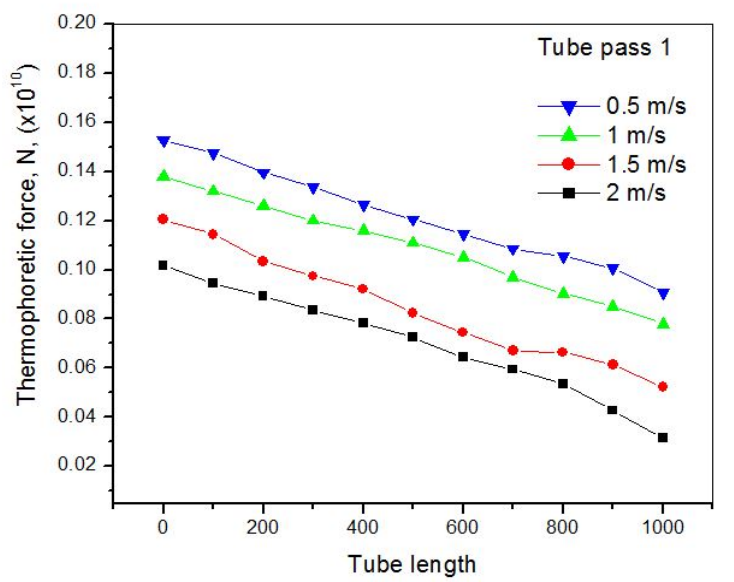

(a)

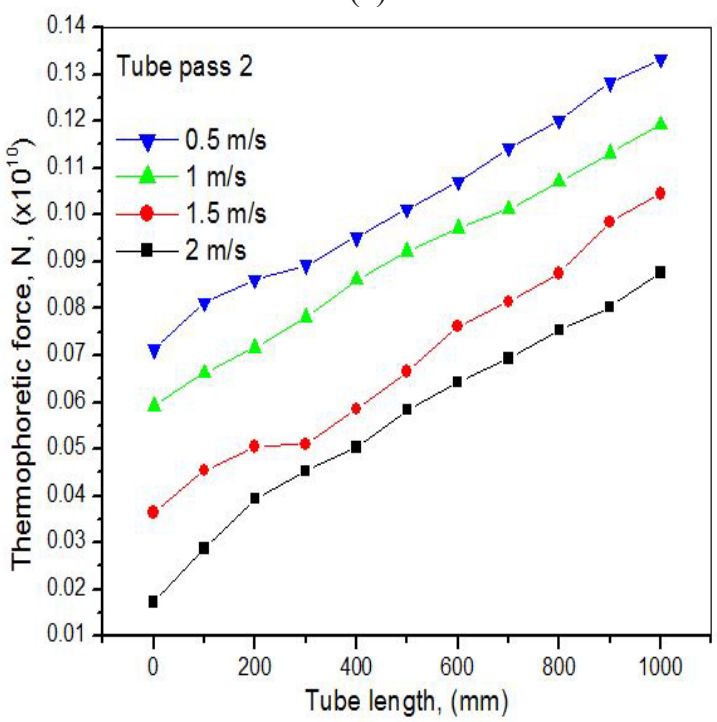

(b)

Fig. 6. Thermophoretic force vs tube length across the tube passes (a) 1 and (b) 2

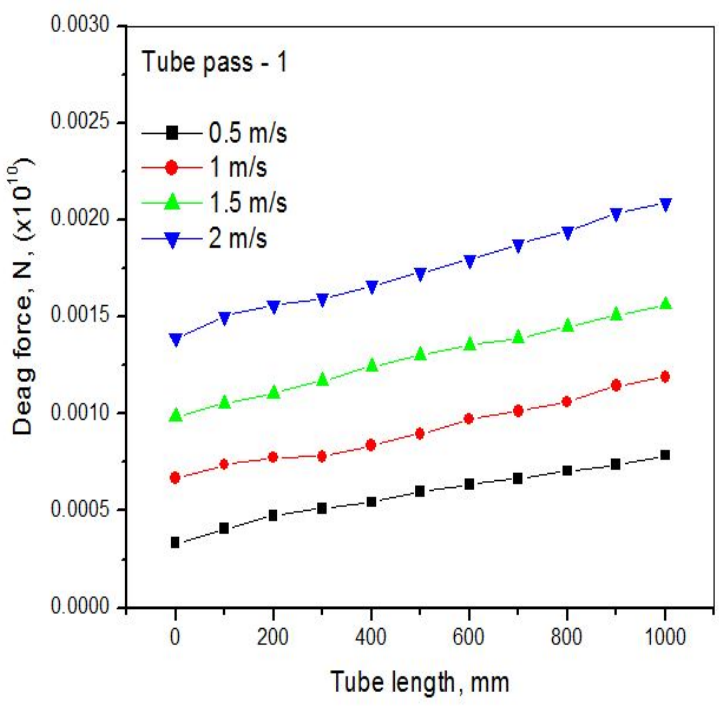

(a) 


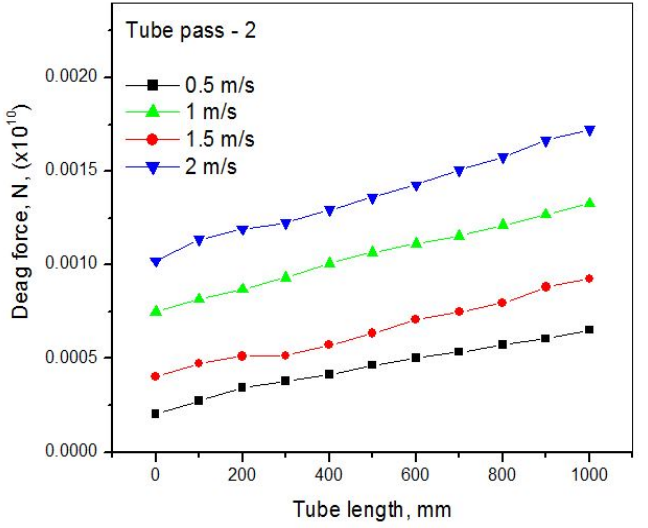

(b)

Fig. 7. Drag force vs tube length across the tube passes (a) 1 and (b) 2

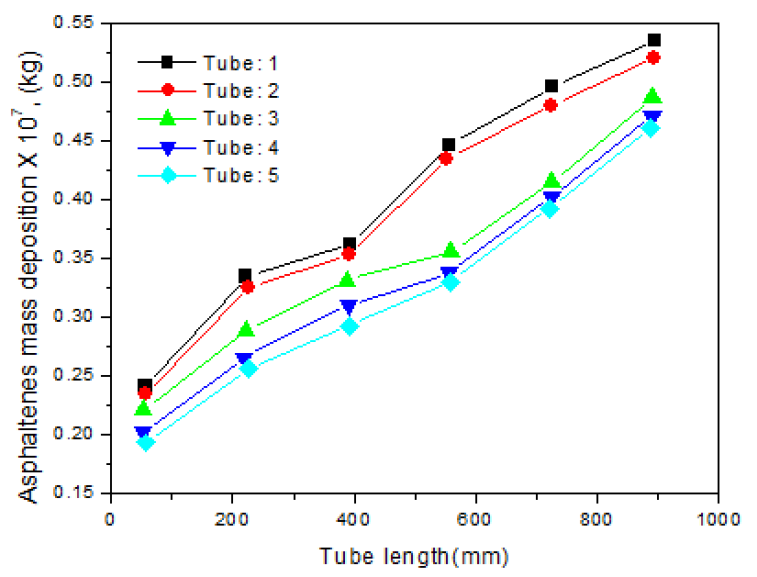

(a)

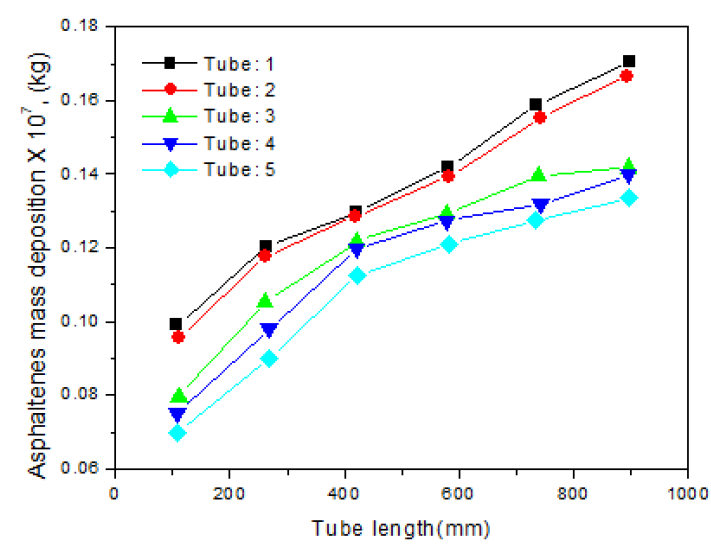

(b)

Fig. 8. Asphaltenes particles velocity distribution in rear header at $60 \mathrm{~s}$ flow time across the tube passes (a) 1 and (b) 2

The gravitational force in tube pass-1 is higher compared with tube pass-2, therefore, the deposition of asphaltenes particles is higher in tube pass compared with tube pass-2. The thermophoretic force (proportional to temperature gradient) is higher in tube pass- 1 compared with tube pass- 2 . As the asphaltenes particles are heated, they acquire high kinetic energies and create a fast flow away from the heat transfer surface, down the temperature gradient between the bulk and the wall. Therefore, the asphaltenes deposition is less in tube pass- 2 compared with tube pass-1. The lift force in the tube pass- 1 is higher compared with tube pass-2, therefore, particles attain a shear force to carry forward with the flow and therefore observed less deposition in tube pass-2.

\section{Conclusions}

Computational Fluid Dynamics simulations provided an insight on the fluid flow behaviour, particles transportation and deposition on the heat transfer surfaces which is certainly impossible to obtain the visibility in the heat exchangers experimentally. The developed CFD methodology analysed the nonuniformity of fouling through particulate fouling routes of asphaltenes in heat exchangers. The susceptible regions of concern through the fouling routes are investigated in the heat exchangers. In tube pass 1 , the gravitational and drag forces increased along the tube, the lift and thermophoretic forces decreased along the tube. As the gravitational force is observed to be the dominating force, the asphaltenes deposition along the tube followed the same trend as the gravitational force. In tube pass 2, the gravitational, thermophoretic and drag forces decreased along the tube and the lift force increased along the tube. Among all the forces, the gravitational force acting on the asphaltenes particles is observed to be high, the drag and thermophoretic forces are observed to have less impact on the asphaltenes particles. As the gravitational force is observed to be the dominating force, the asphaltenes deposition along the tube followed the same trend as the gravitational force.

\section{References}

1. Kazi, S. N. Heat Exchangers-Basics Design Applications. InTech., (2012)

2. Coletti, F. and G. Hewitt, Crude oil fouling: deposit characterization, measurements, and modeling: Gulf Professional Publishing. (2014)

3. Wilson, D.I., Ishiyama, E.M., Paterson, W.R. and Watkinson, A.P., Proceedings of International Conference on Heat Exchanger Fouling and Cleaning VIII, (2009)

4. Qi, G. and F. Jiang, Desalination, 367, 112-125, (2015)

5. Barati, R, Neyshabouri, S.A.A.S. and Ahmadi, G., International Journal of Sediment Research, 33 (4), (2018)

6. Bialik, R.J., Journal of Hydraulic Research, 49 (5), 697-701, (2011)

7. Bialik, R.J., Journal of Hydraulic Research, 49 (1), 23-31, (2011)

8. Bialik, R.J. and M. Karpiński, Journal of Hydraulic Research, 56 (4), 1-7, (2018)

9. Chen, Y, Bai, Y. and Xu, D., International Journal of Sediment Research, 32 (1), 53-59, (2017)

10. Bialik, R.J, Nikora, V.I. and Rowiński, P.M., Acta Geophysica, 60 (6), 1639-1660, (2012) 
11. Shi, H. and X. Yu, International Journal of Sediment Research, 30 (4), 361-370, (2015)

12. Chang, K.-C., Proceedings-National Science Council Republic of China Part a Physical Science and Engineering, 24 (6), 413-421, (2000)

13. Junfeng Yang International Journal of Heat and Mass Transfer, 159, 1-5, (2020)

14. Mario A. Cucumo, Antonio Galloro, Nicoletta Greco, Marilena Mele, Francesco Nicoletti, Diego Perrone, Italian Journal of Engineering Science, 63, 151-157, (2019)

15. Emani, S., Ramasamy, M., \& Shaari, K. Z. K. Applied Thermal Engineering, 149, 105-118, (2019) 